\title{
Mid-term outcomes of endovascular treatment and risk factors for recurrence in patients with Trans-Atlantic-Inter-Society II C/D femoropopliteal lesions
}

\author{
Yisheng $\operatorname{Lin}^{1,2 \#}$, Weihao $\mathrm{Li}^{2 \#}$, Wendao $\mathrm{Liu}^{3}$, Min $\mathrm{Liu}^{2}$, $\mathrm{Yin}^{4}{ }^{4}$, Yong Chen ${ }^{1}$ \\ ${ }^{1}$ Department of Interventional Radiology, Nanfang Hospital, Southern Medical University, Guangzhou, China; ${ }^{2}$ Department of Interventional \\ Radiology, The First Affiliated Hospital of Guangzhou University of Traditional Chinese Medicine, Guangzhou, China; ${ }^{3}$ Department of \\ Interventional Radiology, Guangdong Province Traditional Chinese Medical Hospital, Guangzhou, China; ${ }^{4}$ Department of Gastrointestinal Surgery, \\ The First Affiliated Hospital, Sun Yat-sen University, Guangzhou, China
}

"These authors contributed equally to this work.

Correspondence to: Yin Li. Department of Gastrointestinal Surgery, The First Affiliated Hospital, Sun Yat-sen University, No. 58, Second Zhongshan Road, Guangzhou 510080, China. Email: liyin2736@163.com; Yong Chen. Department of Interventional Radiology, Nanfang Hospital, Southern Medical University, No. 1838 Guangzhou Northern Avenue, Guangzhou 510515, China. Email: cheny102@163.com.

Background: The purpose of this study was to determine the efficacy of interventional endovascular treatment (EVT) and the risk factors for postoperative restenosis in patients with Trans-Atlantic InterSociety Consensus (TASC) II C/D femoropopliteal lesions.

Methods: A total of 55 patients who received EVT for TASC II C/D femoropopliteal lesions (64 affected limbs) from October 2014 to September 2017 were examined. The mean lesion length was 19.6 \pm 5.3 (range, 15.5-26.4 cm). The femoropopliteal lesions were classified as TASC II C and TASC II D in 39 and 16 cases, respectively. The ankle-brachial index $(\mathrm{ABI})$, primary patency rate, secondary patency rate, and limb salvage rate were monitored in follow-up evaluations for up to 24 months. A Cox regression model was used to evaluate the correlation between each of these factors and the restenosis rate after EVT.

Results: Patent TASC II C/D femoropopliteal lesions were present in 59 of the 64 limbs. The mean ABI values for the dorsal pedal artery and posterior tibial artery increased 1 month after treatment from a baseline level of $0.35 \pm 0.12$ to $0.89 \pm 0.10$ and from $0.43 \pm 0.15$ to $0.90 \pm 0.13$, respectively $(\mathrm{P}<0.01)$. The mean follow-up time was 19.3 (range, 6-24) months. The cumulative primary patency rates at 1, 3, 6, 12, 18, and 24 months were $98.3 \%, 91.5 \%, 84.3 \%, 61.1 \%, 53.1 \%$, and $31.1 \%$, respectively. The secondary patency rates at 12 and 24 months were $70.4 \%$ and $60.0 \%$, respectively. Factors with a high hazard ratio included male sex, TASC II $\mathrm{D}$, smoking, and diabetes mellitus (DM).

Conclusions: EVT had a safe and satisfactory mid-term therapeutic effect on TASC II C/D femoropopliteal lesions. Male sex, TASC II D (compared to TASC II C), smoking, and DM were risk factors for restenosis. EVT has a secondary patency rate comparable to that of open surgery and can be considered a first-line treatment for TASC II C/D femoropopliteal lesions.

Keywords: Endovascular treatment (EVT); femoropopliteal lesions; patency rates; recurrence; risk factors

Submitted Feb 07, 2020. Accepted for publication Nov 26, 2020.

doi: 10.21037/qims-20-221

View this article at: http://dx.doi.org/10.21037/qims-20-221 


\section{Introduction}

Peripheral artery disease (PAD) is a manifestation of systemic atherosclerosis in the peripheral arteries, especially those in the lower and upper extremities (1). PAD in the lower extremities mainly manifests as atherosclerosis between the distal abdominal aorta and the lower extremity arteries, resulting in intimal thickening, stenosis, or occlusion. These lesions can cause chronic peripheral ischemia in affected limbs, which can progress to intermittent claudication and ischemic rest pain, which in turn can result in tissue loss and limb-threatening gangrene (2). Treatment options for PAD of the lower extremities include (2) etiology management, moderate exercise, medical therapy, surgical reconstruction, endovascular treatment (EVT), hybrid revascularization, and other advanced therapies. Notably, EVT includes endovascular interventional techniques, such as balloon dilation, stent implantation, and directional atherectomy. The TransAtlantic Inter-Society Consensus (TASC) II guidelines (2) define TASC II C lesions as multiple stenosis or occlusions of more than $15 \mathrm{~cm}$ with or without heavy calcification or recurrent stenosis or occlusions that require treatment after two endovascular interventions. TASC II D lesions are defined as chronic total occlusions of the common femoral artery or superficial femoral artery (involving $>20 \mathrm{~cm}$ of the popliteal artery) or the popliteal artery and proximal trifurcation vessels, respectively.

Surgical revascularization has been recommended as the treatment of choice for TASC II C and D femoropopliteal lesions. However, in clinical practice, many patients with long-segment occlusion lesions are at risk of greater surgical trauma and have limited autologous venous tissue available. Additionally, these patients often show significant surgical contraindications, such as coronary heart disease and cerebral infarction, that increase the risks associated with surgical revascularization. Numerous clinical studies have shown that EVT is effective in treating TASC II C and D lesions (3-9). However, while EVT can successfully restore blood flow and improve clinical symptoms, studies have not systematically assessed the correlations between postoperative recurrence and potential risk factors, such as sex, age, smoking, underlying diseases [e.g., diabetes mellitus (DM)], the severity of ischemic symptoms, and TASC classification. This study sought to evaluate the efficacy of EVT in the treatment of long-segment TASC II C and D femoropopliteal lesions and identify the factors that correlate with restenosis after EVT.

\section{Methods}

\section{Patient population}

The participants in this study were retrospectively selected from patients who had attended The First Affiliated Hospital and The Second Affiliated Hospital of Guangzhou University of Chinese Medicine from October 2014 to September 2017 and were followed up with until September 2019. The study was approved by the Institutional Ethics Board of The First Affiliated Hospital and The Second Affiliated Hospital of Guangzhou University of Chinese Medicine, and individual consent for this retrospective analysis was waived. To be eligible to participate in the study, the patients had to meet the following inclusion criteria: (I) conform to the PAD diagnostic standard; (II) have been classified with Rutherford stage 3 or greater; (III) show moderate to severe intermittent claudication; (IV) have experienced ischemic rest pain; (V) have ulcers or gangrene affecting an arterial vessel in a lower extremity; (VI) have had the TASC II C/D femoropopliteal lesions diagnosed by color Doppler ultrasound, computed tomography angiography (CTA), and/or digital subtraction angiography with a patent distal outflow artery; and (VII) have received prior EVT previously.

\section{$E V T$}

Of the 64 limb lesions, 7 were treated by percutaneous transluminal angioplasty (PTA), and 48 by stenting combined PTA (PTAS). A total of 77 stents were implanted in the 64 limbs (of which, 68 were bare metal stents and 9 were covered stents). Subintimal angioplasty (SIA) was performed in 8 cases and 4 limbs received atherectomies. Interventional EVT was performed using the procedures outlined below.

\section{Puncture approach}

The puncture approach was planned based on the imaging and intraoperative findings. The lesion was accessed via the contralateral femoral artery using a retrograde approach. If necessary, the operator could combine with an anterograde puncture of the ipsilateral femoral artery or retrograde puncture of the ipsilateral peroneal artery (see Figure 1A), anterior tibial artery, dorsalis pedis artery, or popliteal artery, respectively (see Figure $1 B$ ). The extent, degree, outflow tract, and the collateral circulation of the arterial lesions were defined by arteriography. 


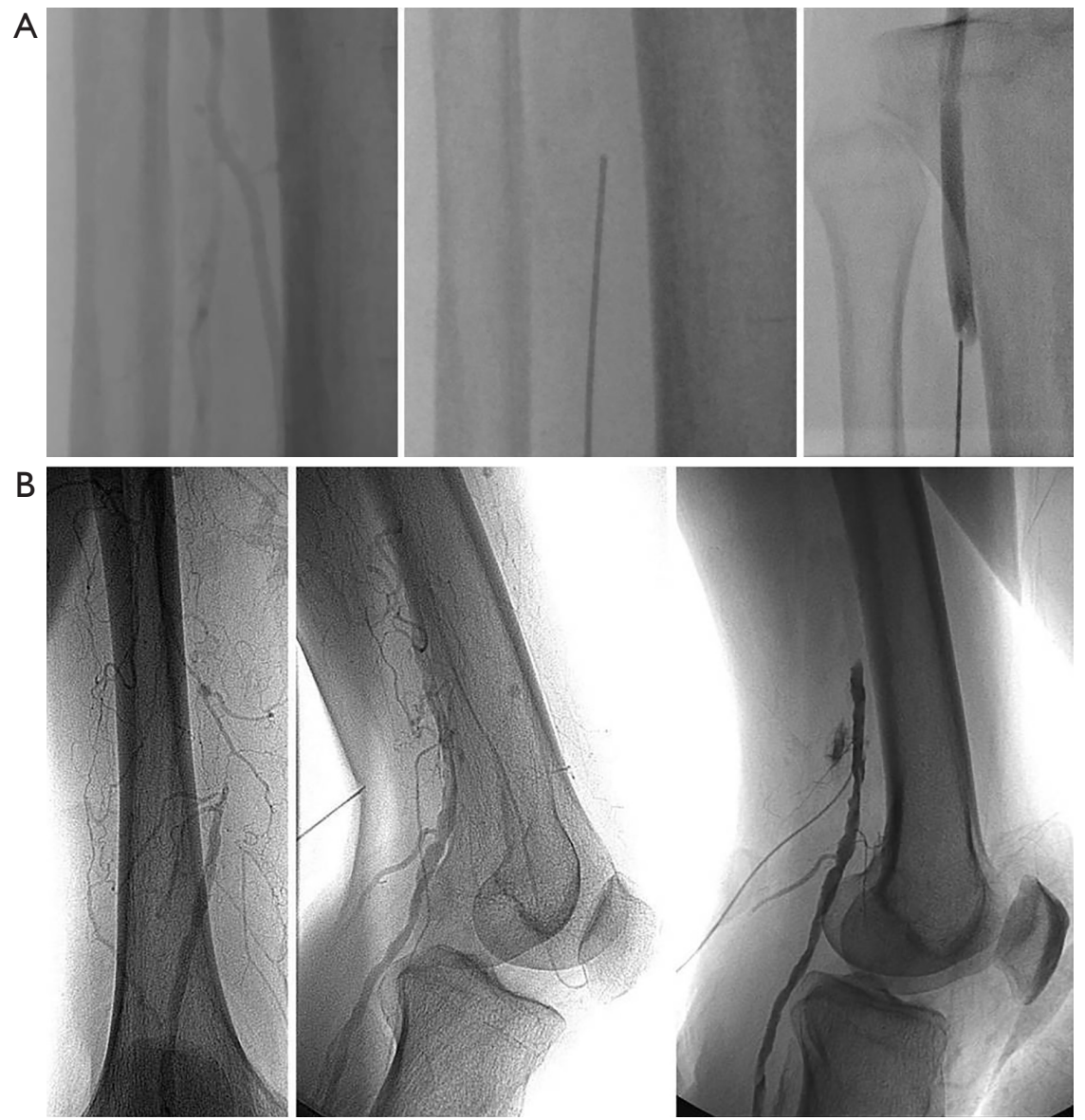

Figure 1 Retrograde puncture of the peroneal artery (A) and popliteal artery (B).

\section{Catbeterization}

A guide wire catheter was adjusted to pass through the true lumen. If the guide wire could not pass through the true lumen, it was inserted in the subintimal space through the lesion intima. SIA (see Figure 2) was required in certain cases to reconstruct the occluded blood supply through a subintimal channel.

\section{Angioplasty}

PTA (Figure 3) was performed. Balloon dilatation was routinely performed for superficial femoral artery lesions. If residual stenosis of $>30 \%$ or subsequent arterial dissection after balloon dilation was observed, endovascular stenting was performed (Figure 4). The patients in this study received self-expanding stents, of which 64 were bare metal stents (LifeStent Flexstar; Bard Peripheral Vascular Inc., Tempe, AZ, USA; Zilver Flex, Cook Medical,
Bloomington, IN, USA) with some stent grafts (Viabahn; Gore Medical, Flagstaff, AZ, USA). Once the guidewire catheter had passed through the true vascular cavity, a percutaneous directional atherectomy was performed (see Figure 5). A peripheral plaque excision system (SilverHawk or Turbohawk) was used to cut through the entire lesion along the preceding $0.035 \mathrm{~cm}$ guide wire, which was passed through the occluded segment into the distal outflow tract using the exchange technique. Guided by the roadmap, the cutting edge was aimed at the sclerosis plaque and pushed to excise the plaque slowly and uniformly.

\section{Criteria for the termination of treatment}

Procedural success was defined as $<30 \%$ of residual stenosis being detected, or at least 1 open artery below the knee joint being confirmed by a repeat angiography after treatment. 



Figure 2 Subintimal angioplasty. (A,B) A guide wire was used to create a subintimal channel. (C) Balloon dilation, (D) the subintimal wire capture method, and (E) subintimal arterial flossing with antegrade retrograde intervention (SAFARI).

\section{Drug-assisted treatment}

Heparinization $(3,000-5,000 \mathrm{U})$ was performed intraoperatively. The subcutaneous injection of lowmolecular-weight heparin $(4,000 \mathrm{U})$ was performed twice a day for 5 days as anticoagulant therapy after the intervention. Thereafter, the patient was switched to an oral antiplatelet treatment of aspirin and clopidogrel bisulfate (Plavix, Bristol-Myers Squibb, New York, NY, USA). Clopidogrel was continued for 6 months at $75 \mathrm{mg} /$ day and aspirin was continued indefinitely at $100 \mathrm{mg}$ /day. The coagulation index was regularly reviewed to maintain the international standardized ratio of 2.0-3.0.

\section{Follow-up evaluations}

Patients attended follow-up evaluations at 1, 3, 6, 12, 18, and 24 months. At the evaluations, any improvement in signs and symptoms (intermittent claudication and ischemic rest pain), the recovery of foot ulceration or gangrene, and the healing of any major (above-the-ankle) or minor (belowthe-ankle) amputations were recorded. At the follow-up examinations, the ankle-brachial index (ABI), color Doppler ultrasounds, and/or CTAs of the lower limb arteries were administered. The CTAs were performed at 1, 6, 12, and 24 months.

\section{Follow-up index}

Primary patency was defined as a treated lower limb vessel that remained patent without any additional PTA. In addition, primary patency was indicated where no stenosis or occlusion $>50 \%$ was observed in the color Doppler ultrasounds, CTAs, or digital subtraction angiographies. 

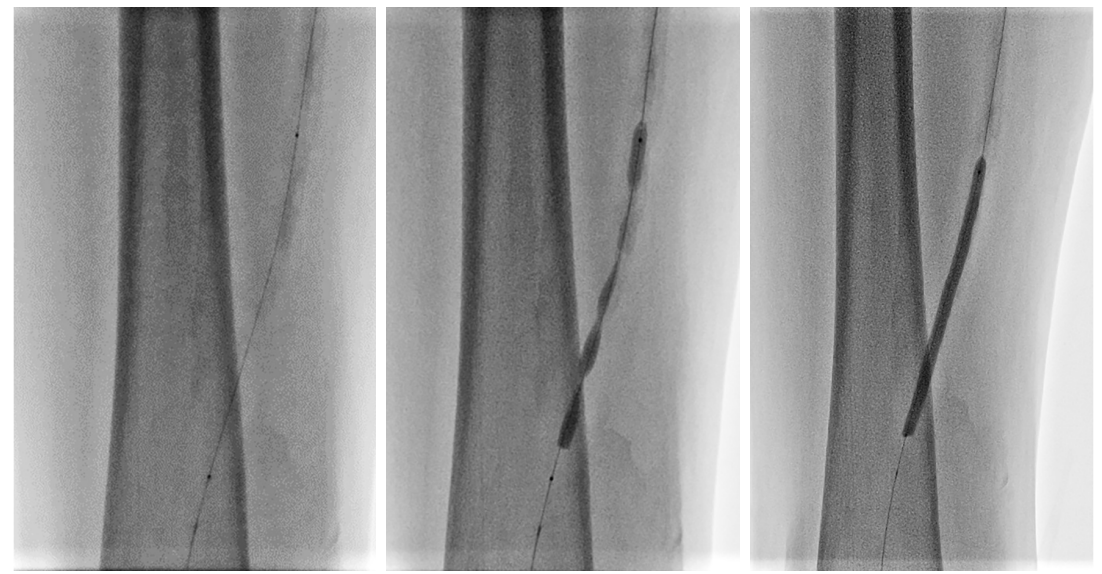

Figure 3 Percutaneous transluminal angioplasty.
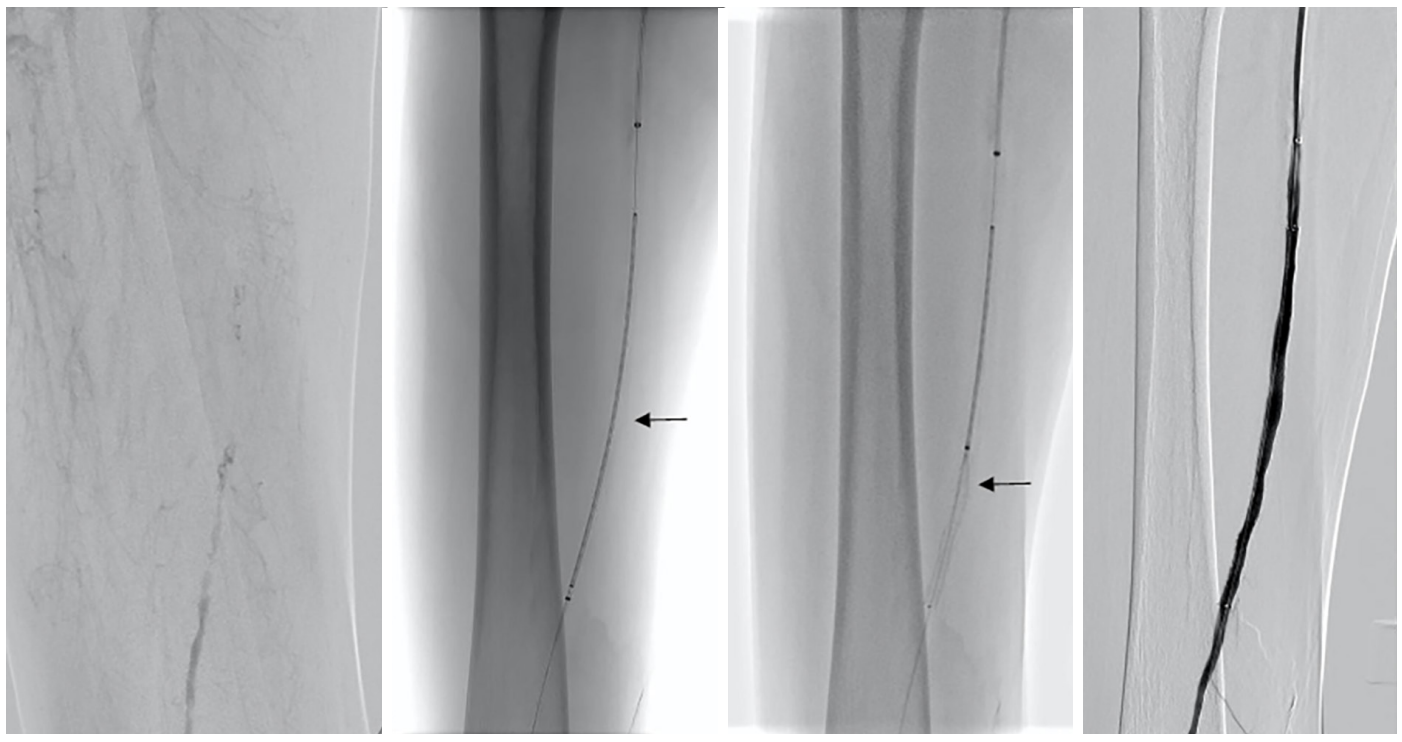

Figure 4 Intravascular stent implantation (black arrows).

Secondary patency was defined as a treated vessel that subsequently became stenosed or occluded during the follow-up evaluations, but, following reintervention, then reopened and remained reopened until the end of the follow-up period. Arterial restenosis was defined as stenosis $>50 \%$ or color Doppler peak systolic blood flow velocity $>2$ times the reference value. Arterial occlusion was defined as no blood flow in arterial lumen according to color Doppler ultrasounds or CTAs. The limb salvage rate included limbs that did not require major amputation, the healing of unavoidable transmetatarsal or toe amputation, and the recovery of foot ulcers after PTA. According to the "Recommended Scale for Evaluation of Postoperative Clinical Status" proposed by Rutherford et al. (10) in 1997, the outcome grade could be determined according to the clinical symptoms and the ABI improvement. No change in clinical status was scored as 0 points; clinical status improvement was scored on a scale of +1 to +3 points; and clinical status deterioration was scored on a scale of -1 to -3 points.

\section{Statistical analysis}

Continuous variables are expressed as the mean \pm standard 

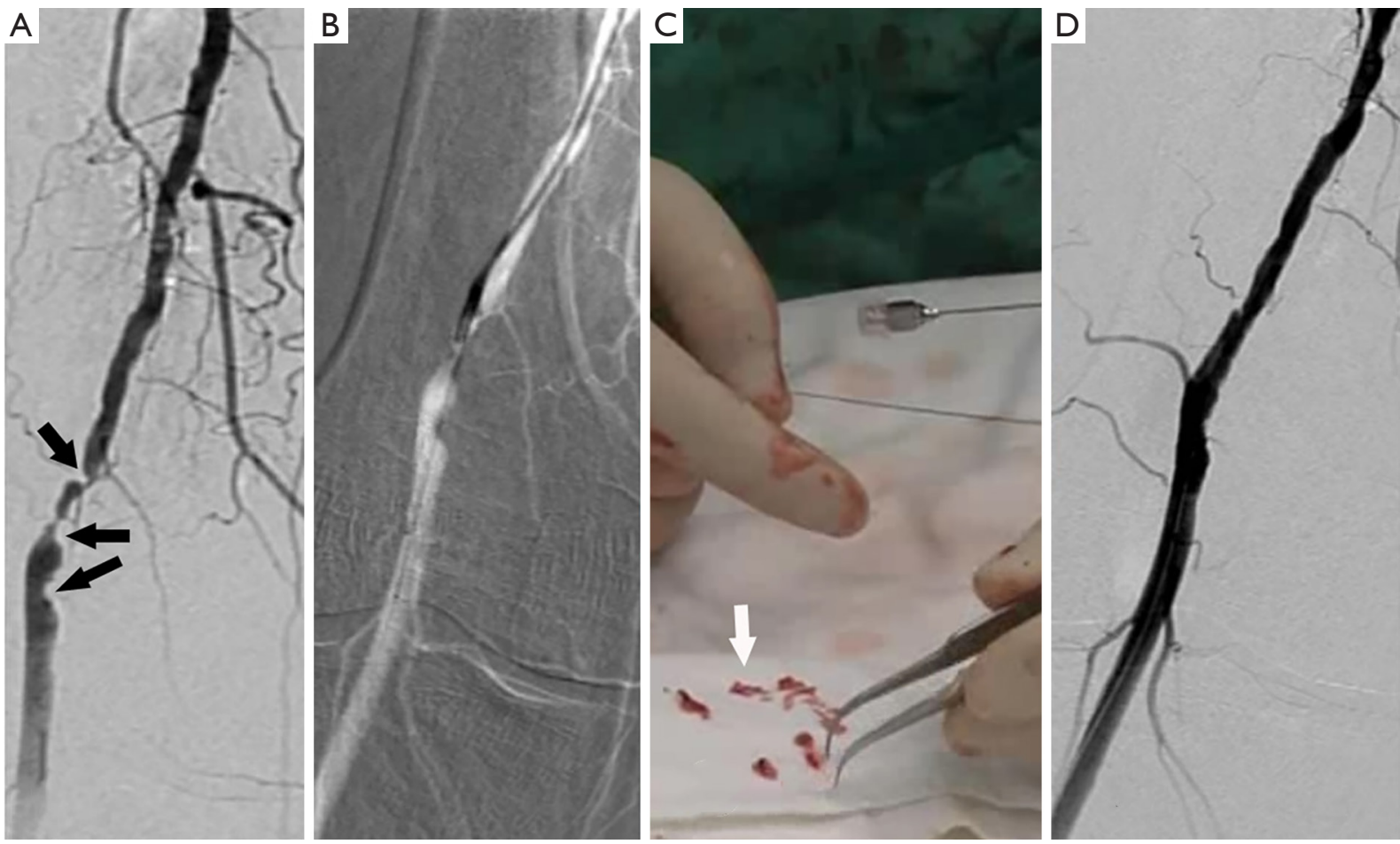

Figure 5 Directional atherectomy. (A) Popliteal artery plaque (black arrows), (B) a directional atherectomy with a TurboHawk device, (C) removed plaque tissue (white arrow), and (D) the use of a repeat angiography showing good recanalization after angioplasty.

deviation. A paired design data t-test was used to compare the ABIs of the affected limbs before the procedure, after the procedure, and during the follow-up evaluations. The two-component variables are presented as percentages. Continuous and binary variables were evaluated separately using Fisher's exact test. The patency and limb-salvage rates were analyzed using the Kaplan-Meier method. A Cox regression model was used to evaluate the correlations between various factors and post-operative recurrence. Data management and all statistical analyses were performed using SPSS for Windows (version 22.0; IBM Corp., Armonk, NY, USA). A P value $<0.05$ was considered statistically significant.

\section{Results}

Ultimately, 55 patients ( 34 males) with TASC II C/D femoropopliteal lesions who met the inclusion criteria were enrolled in the study. Of these, 39 had C lesions (70.9\%) and 16 had D lesions (29.1\%). Lesions were observed in patients' left lower limbs $(\mathrm{n}=29)$, right lower limbs $(\mathrm{n}=23)$, and both lower limbs $(\mathrm{n}=6)$. In total, 64 limbs were treated. The mean age of the participants was $73 \pm 9.5$ (range, $47-82$ ) years.
The mean pretreatment $\mathrm{ABI}$ of the affected dorsal arteries and the posterior tibial arteries was $0.35 \pm 0.12$ (range, $0-0.63$ ) and $0.43 \pm 0.15$ (range, $0-0.72$ ), respectively. The patient demographic and clinical data are shown in Table 1. All 64 limbs of the 55 patients were treated with an interventional therapy. Following confirmation by angiographies, 59 limbs (92.2\%) were recanalized after the interventional treatments. The Rutherford scales of the patients are shown in Table 2. Bypass surgery was performed on 5 limbs $(7.8 \%)$ following unsuccessful interventional therapy. One month after the interventional therapy, the mean ABI increased significantly to $0.89 \pm 0.10$ (range, $0-1.07$ ) and $0.90 \pm 0.13$ (range, $0-1.16$ ) in the dorsal pedal arteries and the posterior tibial arteries, respectively $(\mathrm{P}<0.01)$. The mean follow-up period was 19.3 months (range, 6.0-24.0 months) (see Table 3). The primary patency rates at $1,3,6,12,18$, and 24 months after interventional therapy were $98.3 \%, 91.5 \%, 84.3 \%, 61.1 \%, 53.1 \%$, and $31.1 \%$, respectively (Figure 6). The secondary patency rates at 12 and 24 months were $70.4 \%$ and $60.0 \%$, respectively (Figure 7). The limb salvage rates were $94.4 \%$ and $86.7 \%$ at 12 months and 24 months, respectively (Figure 8). The patency and salvage rates for the patients who received 
Table 1 Demographic and clinical characteristics of the study participants

\begin{tabular}{lc}
\hline Variable & $\mathrm{n}=55(\%)$ \\
\hline Male & $34(61.8)$ \\
Long history of smoking (>10/day, >10 years) & $22(40.0)$ \\
Arterial hypertension & $49(89.1)$ \\
Diabetes mellitus & $35(63.6)$ \\
Hyperlipoproteinemia & $26(47.3)$ \\
Coronary artery disease & $17(30.9)$ \\
Cerebrovascular disease history & $19(34.5)$ \\
Chronic renal failure Rutherford classification & $6(10.9)$ \\
0-2 & 0 \\
3. Severe IC & $15(27.3)$ \\
4. Rest pain & $22(40.0)$ \\
5. Mild tissue loss (mild ulcer) & $10(18.2)$ \\
6. Severe tissue loss (ulcer or gangrene) & $8(14.5)$ \\
\hline
\end{tabular}

IC, intermittent claudication.

Table 2 Postoperative clinical status evaluation

\begin{tabular}{lcc}
\hline $\begin{array}{l}\text { Grade of clinical presentation } \\
\text { and ABI improvement }\end{array}$ & $\mathrm{n}=64(\%)$ & $\begin{array}{c}\text { Total improvement } \\
(\mathrm{n}=64)(\%)\end{array}$ \\
\hline +3: markedly improved & $35(54.7)$ & $59(92.2)$ \\
+2 : moderately improved & $18(28.1)$ & \\
+1: minimally improved & $6(9.4)$ \\
0: no change & $3(4.7)$ \\
-1: mildly worse & $1(1.6)$ \\
-2: moderately worse & $0(0)$ \\
-3: markedly worse & $1(1.6)$ \\
\hline
\end{tabular}

$\mathrm{ABI}$, ankle-brachial index. covered stent grafts $(\mathrm{n}=9)$, SIAs and bare metal stents $(\mathrm{n}=8)$, and directional atherectomies $(n=4)$ are shown in Table 4 . The residual stenosis observed at the narrowest part of the affected arteries was $<30 \%$ of all 59 limbs following EVT (see Figure 9). During follow-up evaluations, 31 patients were diagnosed with $>50 \%$ restenosis or re-occlusion. EVT was repeated on the 24 affected limbs of these patients. Acute thrombosis was observed in 2 cases (see Figure 10). One of these cases was recanalized after thrombolysis, and the other was recanalized after combined thrombolysis and balloon expansion. Eight cases of restenosis were recanalized by balloon dilation, and 13 cases were recanalized by balloon dilation and stent implantation. The hazard ratios for various risk factors for restenosis were determined using a Cox proportional hazards model (Table 5). The hazards ratios for male vs. female, TASC II D vs. C, smoking vs. non-smoking, and DM vs. non-DM were 1.91 $(\mathrm{P}<0.01), 1.69(\mathrm{P}<0.01), 1.73(\mathrm{P}<0.05)$, and $1.23(\mathrm{P}<0.05)$, respectively.

\section{Discussion}

With the development of interventional techniques, advances in EVT have gradually improved the indications of PAD. Recently, TASC I B and C lesions were redefined as TASC II A and B lesions, and the recommended treatment was changed from conventional surgery to EVT. Thus, it is possible to perform EVT on TASC II C/ $\mathrm{D}$ lesions $(2,11)$. In our study, the secondary patency rate after EVT for TASC II C/D femoropopliteal lesions at 12 and 24 months were $70.4 \%$ and $60.0 \%$, respectively, and the salvage rates were $94.4 \%$ and $86.7 \%$, respectively. The efficacy of EVT showed improvement compared to that of surgical treatments, such as bypass surgery $(12,13)$. A meta-analysis showed that the primary patency rate of covered stent graft implantation was higher than that

Table 3 Patency and salvage rates for affected limbs after intervention

\begin{tabular}{|c|c|c|c|c|}
\hline Time (months) & Follow-up rate (\%) & Primary patency rate $(\%)$ & Secondary patency rate $(\%)$ & Limb salvage rate $(\%)$ \\
\hline 1 & $100(59 / 59)$ & $98.3(58 / 59)$ & & $100(59 / 59)$ \\
\hline 3 & $100(59 / 59)$ & $91.5(54 / 59)$ & & $100(59 / 59)$ \\
\hline 6 & $96.6(57 / 59)$ & $84.3(48 / 57)$ & & $100(57 / 57)$ \\
\hline 12 & $91.5(54 / 59)$ & $61.1(33 / 54)$ & $70.4(38 / 54)$ & $94.4(51 / 54)$ \\
\hline 18 & $83.1(49 / 59)$ & $53.1(26 / 49)$ & & $89.8(44 / 49)$ \\
\hline 24 & $76.3(45 / 59)$ & $31.1(14 / 45)$ & $60.0(27 / 45)$ & $86.7(39 / 45)$ \\
\hline
\end{tabular}





Figure 6 Cumulative primary patency survival as analyzed by the Kaplan-Meier method.

of bare metal stents. Compared with bare metal stents, patients who had covered stent graft implantations had a lower need for reinterventions and better improvement in ABI scores (14). Due to the small number of studies and the limited information available, no multivariate analysis was conducted to evaluate the relationship between the TASC II lesion type and the stent type in patency. In this study, a total of 9 covered stents were implanted in the covered stent grafts group. After stratified statistics, the primary and secondary patency rates of the covered stent grafts group at 12 and 24 months were higher than those of the bare metal stent group. Thus, the implantation of covered stent grafts appears to be beneficial to the maintenance of vascular patency after interventional treatments.

Four of the patients in our study were successfully recanalized with percutaneous directional atherectomy devices. Only 1 patient required an additional stent implantation. Stavroulakis et al. (15) and Ibáñez et al. (16) reported a risk of distal arterial embolization as a result of plaque fragments after directional atherectomy, and recommended the use of an embolic protection device (EPD). An EPD was only used in 2 affected limbs, 1 of

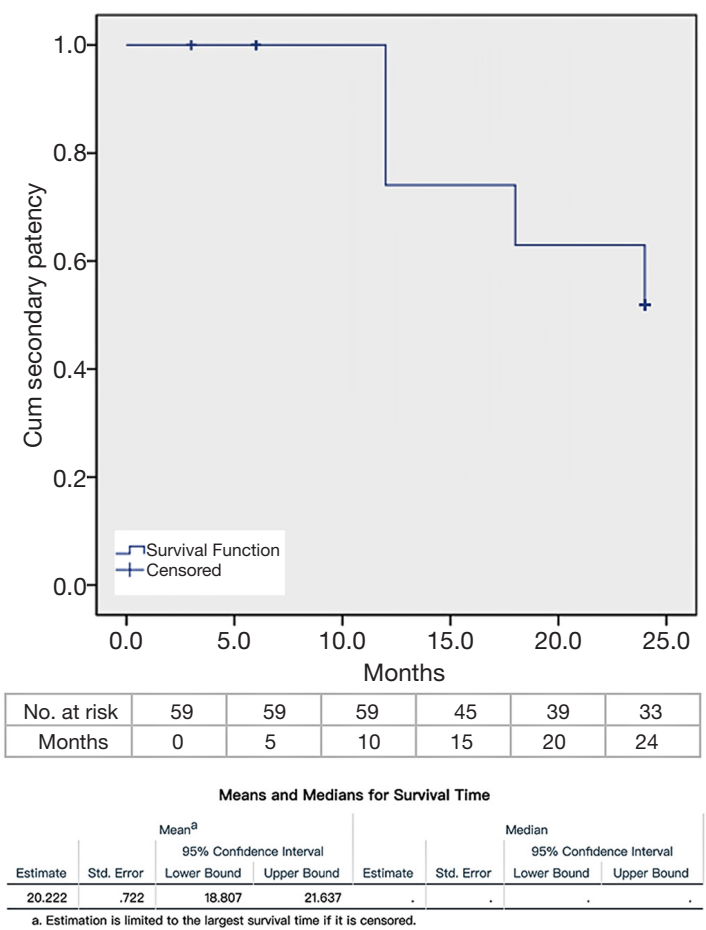

Figure 7 Cumulative secondary patency survival analyzed by the Kaplan-Meier method.

which collected a small amount of plaque tissue, and none of these 4 patients experienced serious complications, such as vascular wall penetration or distal embolization. At the end of our study, the lower extremity arteries remained patent in 3 patients. Only 1 patient showed restenosis at the 12-month follow-up evaluation and had to be recanalized by balloon dilation. It was not possible to determine if these findings were statistically significant due to the small number of cases. However, the percutaneous directional atherectomy has been shown to be a minimally invasive and safe interventional therapy that achieves a high patency rate and requires a low use of stents $(17,18)$.

A Cox proportional hazards model was used to determine the hazard ratios of various risk factors for restenosis after EVT. Males (compared to females) and TASC II D lesions (compared to TASC II C lesions) had higher hazard ratios for restenosis $(\mathrm{P}<0.01)$. Smoking and $\mathrm{DM}$ were also risk factors for restenosis. The results of the statistical analysis showed that the probability of restenosis was higher in men than in women who underwent EVT for TASC II C/ D femoropopliteal lesions. Similarly, previous research has shown that chronic arterial occlusion is closely associated 


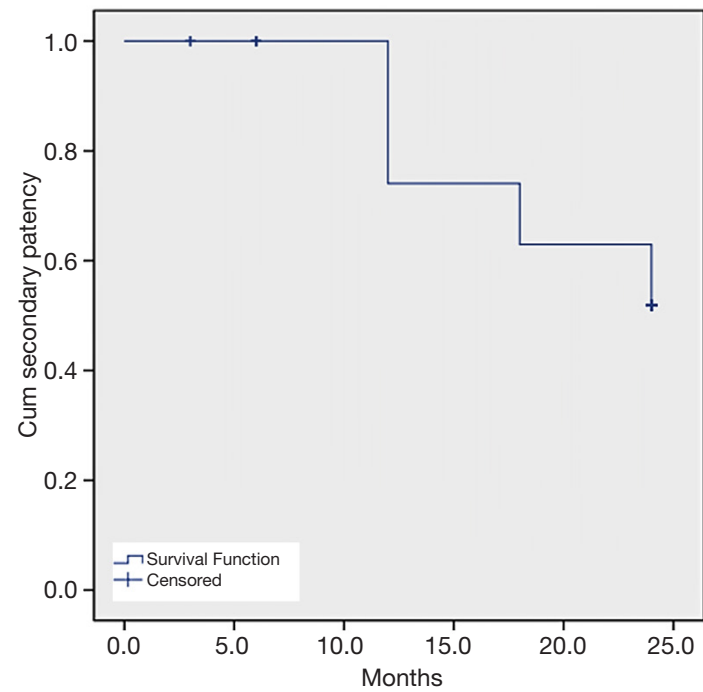

\begin{tabular}{|c|c|c|c|c|c|c|}
\hline No. at risk & 59 & 59 & 59 & 56 & 51 & 45 \\
\hline Months & 0 & 5 & 10 & 15 & 20 & 24 \\
\hline
\end{tabular}

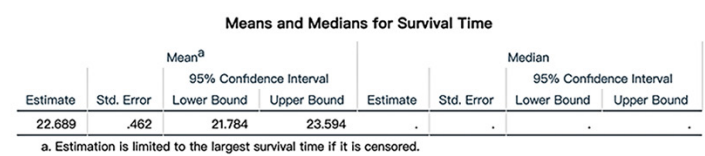

Figure 8 Cumulative salvage rate survival analyzed by the KaplanMeier method. with the impairment of vascular endothelial cells (19). However, while balloon dilation may cause intimal hyperplasia of the arteries, estrogen, which is known to have vasodilatory activity, can protect vascular endothelial cells (20). Women have high estrogen levels; thus, female patients with TASC II C/D femoropopliteal lesions are more likely to maintain vessel patency after EVT. Patients with TASC II D have a larger range of arterial occlusion

Table 4 Patency rate and salvage rate with new technologies and instruments

\begin{tabular}{lcccc}
\hline Therapy & $\begin{array}{c}\text { Follow-up } \\
\text { time } \\
\text { (months) }\end{array}$ & $\begin{array}{c}\text { Primary } \\
\text { patent rate } \\
(\%)\end{array}$ & $\begin{array}{c}\text { Secondary } \\
\text { patency rate } \\
(\%)\end{array}$ & $\begin{array}{c}\text { Limb salvage } \\
\text { rate }(\%)\end{array}$ \\
\hline $\begin{array}{l}\text { Stent graft } \\
\text { group (n=9) }\end{array}$ & 12 & $77.8(7 / 9)$ & $88.9(8 / 9)$ & $100(9 / 9)$ \\
SIA + stent & 12 & $55.6(5 / 9)$ & $66.7(6 / 9)$ & $100(9 / 9)$ \\
group (n=8) & 24 & $50.0(4 / 8)$ & $62.5(5 / 8)$ & $100(8 / 8)$ \\
DA group & 12 & $100(4 / 4)$ & $100(4 / 4)$ & $100(4 / 4)$ \\
$(n=4)$ & 24 & $50.0(2 / 4)$ & $100(4 / 4)$ & $100(4 / 4)$ \\
\hline
\end{tabular}

DA, directional atherectomy; SIA, subintimal angioplasty.
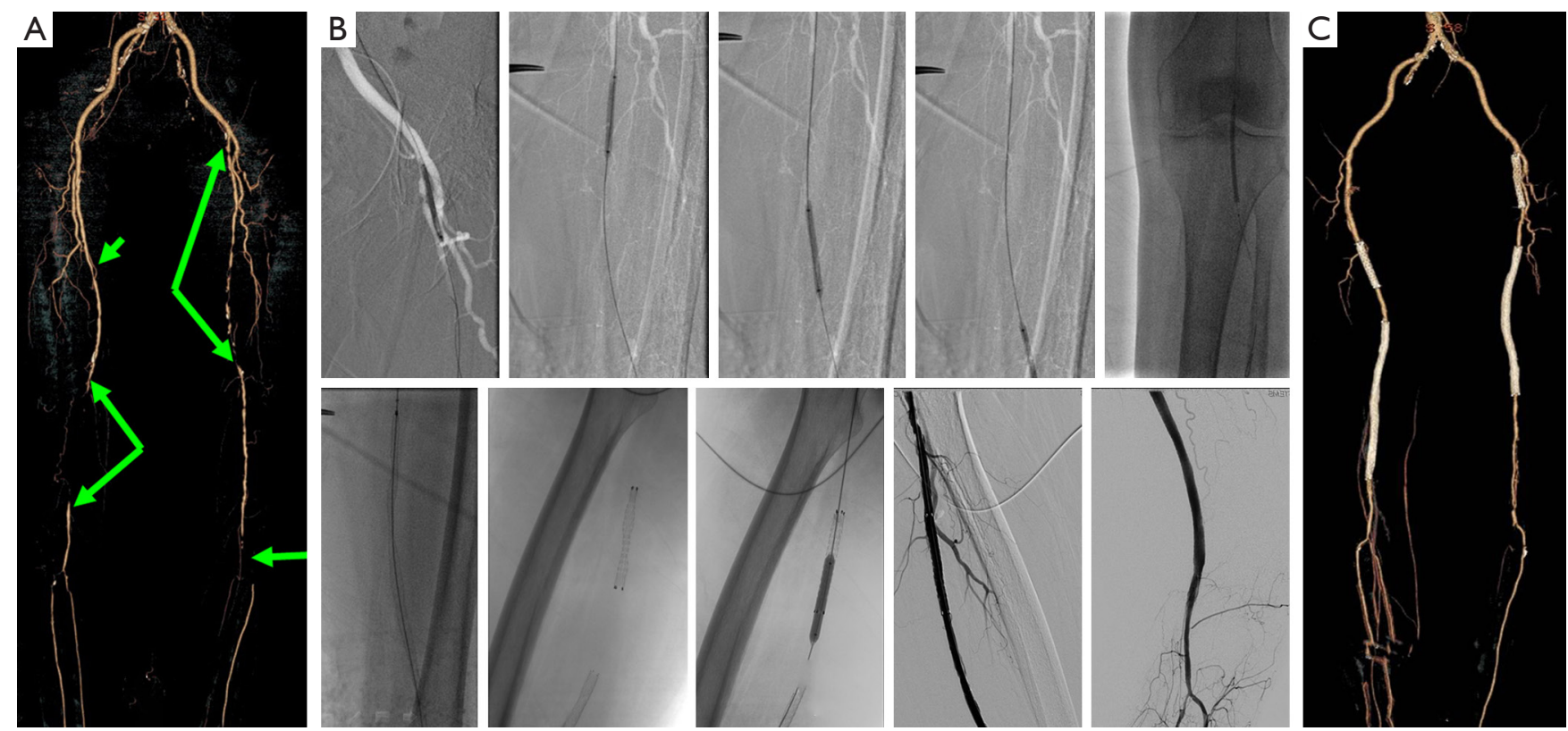

Figure 9 A representative case. (A) Pretreatment computed tomography angiography confirmed TASC II C/D femoropopliteal lesions of both lower limbs (artery stenosis, green arrows). (B) Endovascular treatment, percutaneous transluminal angioplasty and stent implantation, and $(\mathrm{C})$ postoperative computed tomography revealed $<30 \%$ stenosis of the affected arteries. 

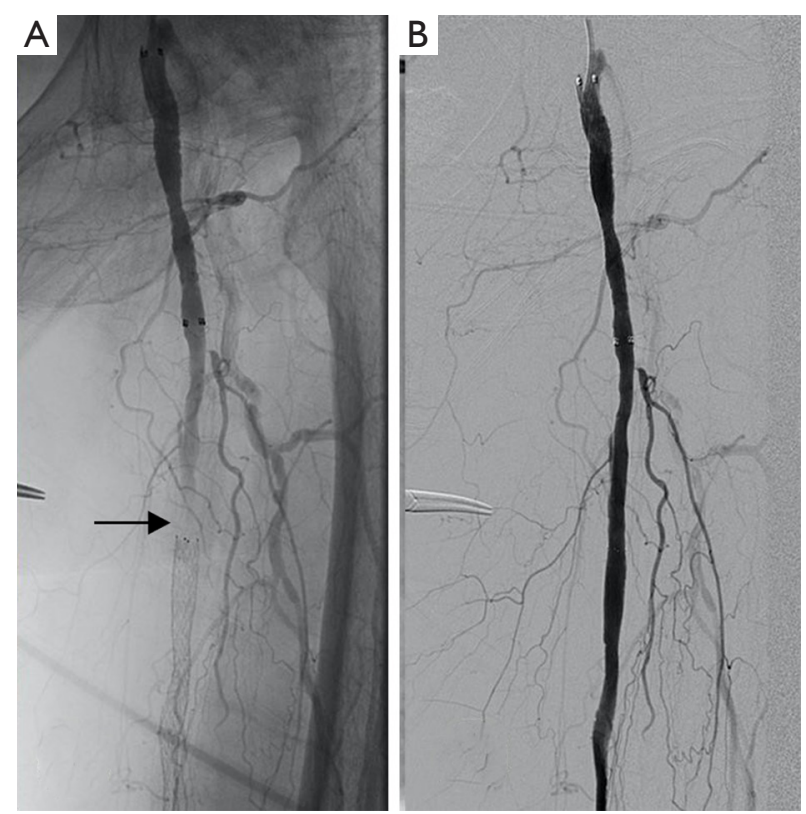

Figure 10 Stent thrombosis. (A) Thrombosis in the stent (black arrow) and (B) recanalization after thrombolysis and percutaneous transluminal angioplasty.

lesions than patients with TASC II C. Additionally, the incidence of restenosis after EVT is higher for TASC II D femoropopliteal lesions than for TASC II C lesions. However, an ongoing prospective study of EVT for TASC II D femoropopliteal lesions reported primary and secondary patency rates of $41 \%$ and $79 \%$ respectively, in the second year following the interventional therapy (4). Accordingly, EVT for TASC II D femoropopliteal lesions in the current study achieved satisfactory symptomatic improvement and secondary patency rates.

In support of the findings of the current study, Olin et al. (21) found that smoking was an independent risk factor of PAD. Smoking increased the risk of PAD by 4 times and increased the risk of amputation in patients with intermittent claudication by $30 \%$. Additionally, for patients with intermittent claudication who continued to smoke after diagnosis, the 5 -year mortality rate could be as high as $40 \%$ to $50 \%$. Conversely, among patients who stopped smoking, the 10-year mortality rate was reduced from 54\% to $18 \%$, and the risk of critical limb ischemia and symptoms of intermittent claudication also decreased (22). Nicotine is the main harmful ingredient in tobacco and damages blood vessels through vascular endothelial injury, formation, rupture or shedding of plaque, and vasospasm $(23,24)$. Tobacco also contains procoagulant substances that can lead
Table 5 Hazards ratios for various risk factors for restenosis

\begin{tabular}{lcc}
\hline Factors & Corrected HR $(95 \% \mathrm{Cl})$ & $\mathrm{P}$ value \\
\hline Male sex & $1.91(1.42-2.29)$ & $<0.01$ \\
Age (per 10 years) & $1.08(0.95-1.30)$ & 0.351 \\
Smoking & $1.73(1.29-2.14)$ & 0.012 \\
Arterial hypertension & $1.02(0.85-1.17)$ & 0.865 \\
Diabetes mellitus & $1.23(1.06-1.45)$ & 0.032 \\
Hyperlipoproteinemia & $1.01(0.80-1.22)$ & 0.837 \\
Chronic renal failure & $0.97(0.91-1.14)$ & 0.864 \\
Critical limb ischemia & $1.12(0.98-1.35)$ & 0.281 \\
TASC II D lesions & $1.69(1.55-2.04)$ & $<0.01$ \\
\hline
\end{tabular}

$\mathrm{Cl}$, confidence interval; $\mathrm{HR}$, hazard ratio.

to a hypercoagulable state. Thus, the risk of restenosis after surgical interventions is significantly higher for smokers than for non-smokers.

Similarly, the occurrence and progression of lower extremity arteriosclerotic occlusion is closely related to the presence and progression of DM. DM can increase the risk of PAD by $15 \%$ to $40 \%$ (6). In patients with diabetes, which is a disorder of blood glucose, fat, and protein metabolism, any vessel walls are prone to atherosclerosis and intimal hyperplasia. Thus, patients with TASC II C/ $\mathrm{D}$ femoropopliteal lesions who show poor glycemic control are more likely to have PAD with restenosis or re-occlusion than those with normal blood glucose levels. However, reasonable revascularization is observed after EVT in the short term. This study indicates that the risk of restenosis in diabetic patients is 1.23 times higher than the risk of restenosis in non-diabetic patients, which is consistent with the results of previous studies $(25,26)$.

Our study had several limitations. First, as it was a retrospective study with a limited number of patients, the statistical power was low. Second, due to medical insurance restrictions, paclitaxel balloon and drug-eluting stents, which have significant effects on patency, could not be used in our study. Third, interventional therapy was not compared with surgical bypass treatment. In the future, large-scale studies that address these limitations should be conducted to improve the reliability of the results.

In conclusion, the effect of EVT on TASC II C/D femoropopliteal lesions was found to be satisfactory and safe during the follow-up period. Following the Cox regression analyses of patients with TASC II C/D femoropopliteal 
disease, we identified male sex, TASC II D lesions, disease, smoking, and DM as risk factors of restenosis. The primary patency rate of EVT was not higher than that of open surgery; however, EVT had a similar secondary patency rate and should be considered a first-line treatment for TASC II $\mathrm{C} / \mathrm{D}$ femoropopliteal lesions.

\section{Acknowledgments}

Funding: This work was funded by the Natural Science Foundation of Guangdong Province (No. 2019A1515012118) and the Foundation of Guangdong Science and Technology Department (No. 2015A020211024).

\section{Footnote}

Conflicts of Interest: All authors have completed the ICMJE uniform disclosure form (available at http://dx.doi. org/10.21037/qims-20-221). All authors report receiving grants from the Natural Science Foundation of Guangdong Province (No. 2019A1515012118), and grants from the Foundation of Guangdong Science and Technology Department (No. 2015A020211024) during the conduct of the study.

Ethics Statement: The study was conducted in accordance with the Declaration of Helsinki (as revised in 2013). The study was approved by the Institutional Ethics Board of The First Affiliated Hospital and The Second Affiliated Hospital of Guangzhou University of Chinese Medicine, and individual consent for this retrospective analysis was waived.

Open Access Statement: This is an Open Access article distributed in accordance with the Creative Commons Attribution-NonCommercial-NoDerivs 4.0 International License (CC BY-NC-ND 4.0), which permits the noncommercial replication and distribution of the article with the strict proviso that no changes or edits are made and the original work is properly cited (including links to both the formal publication through the relevant DOI and the license). See: https://creativecommons.org/licenses/by-nc-nd/4.0/.

\section{References}

1. Hiatt WR, Goldstone J, Smith SC Jr, McDermott M, Moneta G, Oka R, Newman AB, Pearce WH. Atherosclerotic Peripheral Vascular Disease Symposium
II: nomenclature for vascular diseases. Circulation 2008;118:2826-9.

2. Norgren L, Hiatt WR, Dormandy JA, Nehler MR, Harris KA, Fowkes FG; TASC II Working Group. Inter-Society Consensus for the Management of Peripheral Arterial Disease. J Vasc Surg 2007;45:S5-67.

3. Guo X, Xue G, Huang X, Xie H, Liang W, Zhang J, Lin F, Yao T. Outcomes of endovascular treatment for patients with TASC II D femoropopliteal occlusive disease: a single center study. BMC Cardiovasc Disord 2015;15:44.

4. Torres-Blanco Á, Edo-Fleta G, Gomez-Palones F, MolinaNacher V, Ortiz-Monzon E. Mid-Term Outcomes of Endovascular Treatment for TASC-II D Femoropopliteal Occlusive Disease with Critical Limb Ischemia. Cardiovasc Intervent Radiol 2016;39:344-52.

5. Geraghty PJ, Mewissen MW, Jaff MR, Ansel GM. Three-year results of the VIBRANT trial of VIABAHN endoprosthesis versus bare nitinol stent implantation for complex superficial femoral artery occlusive disease. J Vasc Surg 2013;58:386-95.e4.

6. Leopardi M, Houbballah R, Becquemin JP. Effectiveness of Zilver PTX eluting stent in TASC C/D lesions and restenosis. J Cardiovasc Surg (Torino) 2014;55:229-34.

7. Sethi SS, Lee MS. Drug-Coated Balloons for Infrainguinal Peripheral Artery Disease. J Invasive Cardiol 2016;28:281-6.

8. Davaine JM, Azéma L, Guyomarch B, Chaillou P, Costargent A, Patra P, Lambert G, Gouëffic Y. Oneyear Clinical Outcome after Primary Stenting for TransAtlantic Inter-Society Consensus (TASC) C and D Femoropopliteal Lesions (The STELLA "STEnting Long de L'Artère fémorale superficielle" Cohort). Eur J Vasc Endovasc Surg 2012;44:432-41.

9. Elmahdy MF, Buonamici P, Trapani M, Valenti R, Migliorini A, Parodi G, Antoniucci D. Long-Term Primary Patency Rate After Nitinol Self-Expandable Stents Implantation in Long, Totally Occluded Femoropopliteal (TASC II C \& D) Lesions. Heart Lung Circ 2017;26:604-11.

10. Rutherford RB, Baker JD, Ernst C, Johnston KW, Porter JM, Ahn S, Jones DN. Recommended standards for reports dealing with lower extremity ischemia: revised version. J Vasc Surg 1997;26:517-38.

11. Halliday A, Bax JJ. The 2017 ESC Guidelines on the Diagnosis and Treatment of Peripheral Arterial Diseases, in Collaboration With the European Society for Vascular Surgery (ESVS). Eur J Vasc Endovasc Surg 2018;55:301-2.

12. Olinic DM, Spinu M, Olinic M, Homorodean C, Tataru DA, Liew A, Schernthaner GH, Stanek A, Fowkes 
G, Catalano M. Epidemiology of peripheral artery disease in Europe: VAS Educational Paper. Int Angiol 2018;37:327-34.

13. Enzmann FK, Nierlich P, Aspalter M, Hitzl W, Dabernig W, Hölzenbein T, Ugurluoglu A, Seitelberger R, Linni K. Nitinol Stent Versus Bypass in Long Femoropopliteal Lesions: 2-Year Results of a Randomized Controlled Trial. JACC Cardiovasc Interv 2019;12:2541-9.

14. Hajibandeh S, Hajibandeh S, Antoniou SA, Torella F, Antoniou GA. Covered vs Uncovered Stents for Aortoiliac and Femoropopliteal Arterial Disease: A Systematic Review and Meta-analysis. J Endovasc Ther 2016;23:442-52.

15. Stavroulakis K, Bisdas T, Torsello G, Stachmann A, Schwindt A. Combined Directional Atherectomy and Drug-Eluting Balloon Angioplasty for Isolated Popliteal Artery Lesions in Patients With Peripheral Artery Disease. J Endovasc Ther 2015;22:847-52.

16. Ibáñez MA, Cenizo N, Río L, Sánchez A, San Norberto E, Brizuela JA, Gutiérrez V, Vaquero C. Clinical and haemodynamic evolution of lesions treated by means of atherectomy with SilverHawk in the femoropopliteal sector. Eur J Radiol 2011;80:543-7.

17. McKinsey JF, Zeller T, Rocha-Singh KJ, Jaff MR, Garcia LA. Lower extremity revascularization using directional atherectomy: 12 -month prospective results of the DEFINITIVE LE study. JACC Cardiovasc Interv 2014;7:923-33.

18. Minko P, Buecker A, Jaeger S, Katoh M. Three-year results after directional atherectomy of calcified stenotic lesions of the superficial femoral artery. Cardiovasc Intervent Radiol 2014;37:1165-70.

Cite this article as: Lin Y, Li W, Liu W, Liu M, Li Y, Chen Y. Mid-term outcomes of endovascular treatment and risk factors for recurrence in patients with Trans-Atlantic-Inter-Society II C/D femoropopliteal lesions. Quant Imaging Med Surg 2021;11(5):2028-2039. doi: 10.21037/qims-20-221
19. Tasatargil A, Tanriover G, Barutcigil A, Turkmen E. Protective effect of resveratrol on methylglyoxal-induced endothelial dysfunction in aged rats. Aging Clin Exp Res 2019;31:331-8.

20. Aronow WS, Chul A, Weiss MB, Sateesh B. Relation of increased hemoglobin A(1c) levels to severity of peripheral arterial disease in patients with diabetes mellitus. Am J Cardiol 2007;99:1468-9.

21. Olin JW, Sealove BA. Peripheral artery disease: current insight into the disease and its diagnosis and management. Mayo Clin Proc 2010;85:678-92.

22. Sartipy F, Sigvant B, Lundin F, Wahlberg E. Ten Year Mortality in Different Peripheral Arterial Disease Stages: A Population Based Observational Study on Outcome. Eur J Vasc Endovasc Surg 2018;55:529-36.

23. Lu JT, Creager MA. The relationship of cigarette smoking to peripheral arterial disease. Rev Cardiovasc Med 2004;5:189-93.

24. Hanna ST. Nicotine effect on cardiovascular system and ion channels. J Cardiovasc Pharmacol 2006;47:348-58.

25. Al-Khoury G, Marone L, Chaer R, Rhee R, Cho J, Leers S, Makaroun M, Gupta N. Isolated femoral endarterectomy: impact of SFA TASC classification on recurrence of symptoms and need for additional intervention. J Vasc Surg 2009;50:784-9.

26. Lee MS, Rha SW, Han SK, Choi BG, Choi SY, Ali J, Xu S, Ngow HA, Lee JJ, Lee KN, Kim JB, Lee S, Na JO, Choi CU, Lim HE, Kim JW, Kim EJ, Park CG, Seo HS, Kong J, Oh DJ. Comparison of diabetic and non-diabetic patients undergoing endovascular revascularization for peripheral arterial disease. J Invasive Cardiol 2015;27:167-71. 